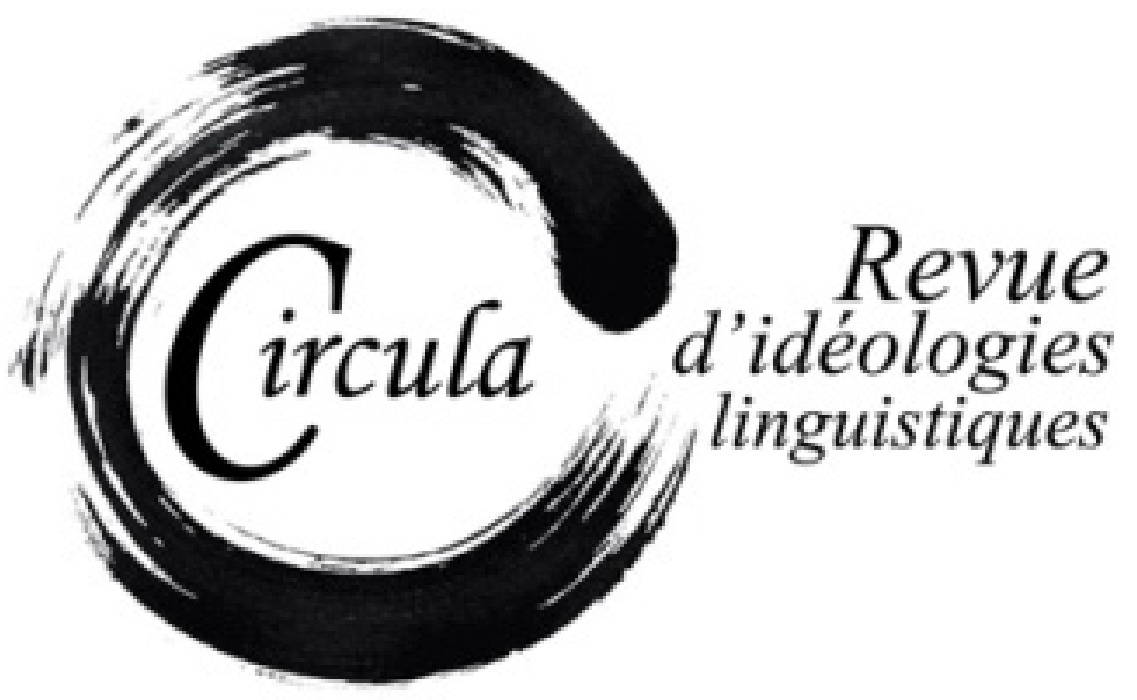

TITRE: IL COMMENTO LINGUISTICO COME TRADIZIONE DISCORSIVA NELLA STAMPA PERIODICA DI METÀ SETTECENTO

Auteur: RAPHAEL MERIDA, UnIVERSITÀ DEGLI STUdI DI MESSINA

Revue: CIRCULA, NUMÉRO 11

PAgES: $46-62$

ISSN: 2369-6761

URI: HTTP://HDL.HANDLE.NET/11143/17840

DOI: HTTPS://DOI.ORG/10.17118/11143/17840 


\section{Il commento linguistico come tradizione discorsiva nella stampa periodica di metà Settecento}

Raphael Merida, Università degli studi di Messina rmerida@unime.it

Riassunto: La pratica del commento linguistico all'interno della stampa periodica comincia a diffondersi prevalentemente nell'Ottocento. Gli articoli di cronaca, le recensioni bibliografiche o le semplici novità, però, offrono uno spunto di riflessione linguistica già all'interno di molti periodici della metà del Settecento. In particolare, polemiche o apologie rivolte al conservatorismo dell'Accademia della Crusca, l'innovazione del lessico specialistico tramite nuove traduzioni di opere straniere e gli influssi dei francesismi nel lessico italiano, permettono ai compilatori delle varie riviste letterarie e delle gazzette di discutere, talvolta ampiamente, dei problemi linguistici dell'epoca. La ricerca quindi si propone di individuare una prima forma di commento linguistico all'interno di alcuni periodici a stampa di metà Settecento.

Parole chiave: commento linguistico, Settecento, periodici, Crusca, lessico tecnico.

Abstract: The practice of linguistic commentary within the periodical press began to spread mainly in the 19th century. The news articles, bibliographical reviews or simple news, however, offer a starting point for linguistic reflection already in many periodicals of the mid-eighteenth century. In particular, controversies or apologies addressed to the conservatism of the Accademia della Crusca, the innovation of the specialized lexicon through new translations of foreign works and the influences of the French into the Italian lexicon, allow the compilers of the various literary magazines and journals to discuss, sometimes widely, of the linguistic problems of the time. The research therefore aims to identify a first form of linguistic commentary within some printed periodicals of the mid-eighteenth century.

Keywords: linguistic commentary, 18th century, press, Crusca, special lexicon. 


\section{Introduzione}

Molti importanti studi hanno messo in luce l'evoluzione del giornalismo dalla fine del Seicento ai giorni nostri e ne hanno esaminato i fenomeni lessicali, sintattici e morfologici, dimostrando quanto sia complessa la struttura di un periodico a stampa: infatti, «non si può parlare del linguaggio giornalistico come di una lingua speciale: il giornale è un coacervo di lingue speciali» (De Mauro, 1976: 510) che rappresentano lo specchio dei linguaggi presenti nella società (cf. Beccaria, 1973: 64). Oltre a configurarsi come modello esemplare per l'analisi linguistica, i giornali si rivelano una fonte preziosa per la ricostruzione del dibattito culturale cominciato nei primi anni del Settecento e costantemente portato avanti nei secoli. Basti citare solo alcuni titoli per rendersi conto dello spessore dei nomi circolanti nelle varie redazioni di giornali, riviste e gazzette. Si prendano, per esempio, la Gazzetta di Milano, sulla quale scrisse anche Parini (cf. Bonomi, 2010: online), oppure la Galleria di Minerva e il più famoso Giornale de' letterati d'Italia, all'interno dei quali si era formato un circolo di intellettuali del calibro di Scipione Maffei o Apostolo Zeno (cf. Di Brazzà, 2012). Ben più note, anche per via della forte personalità dei loro fondatori, le vicende del Caffè e della Frusta Letteraria. Il celebre articolo-manifesto dei fratelli Verri Rinunzia avanti notaio degli autori del presente foglio periodico al Vocabolario della Crusca, che fa parte di uno degli articoli di carattere linguistico del Caffè, rappresenta un punto apicale dell'«avversione settecentesca alla politica linguistica della Crusca» (Giovanardi, 1987: 401) e mostra apertamente l'ideologia linguistica dei due autori. Allo stesso modo l'intera impostazione della Frusta Letteraria nasconde, sotto le vesti di domande e risposte fittizie, il pensiero critico di Giuseppe Baretti². I pochi esempi appena citati sono un'importante testimonianza dell'evoluzione del periodico a stampa: tuttavia, la caratura ideologica che si manifesta attraverso le figure di spicco di intellettuali famosi fa sì che l'interesse alla lingua faccia parte del fermento culturale di quel periodo.

Facendo riferimento al concetto di «tradizione discorsiva» di Koch/Oesterreicher (1990: 190-198), ci siamo chiesti se la pratica del commento linguistico, inteso come genere giornalistico autonomo, abbia avuto origine nel giornalismo settecentesco. Gli spunti per questa riflessione teorica derivano dagli studi che negli ultimi anni si sono concentrati sulle cronache linguistiche all'interno dei periodici a stampa (e ultimamente anche nei quotidiani in rete). Infatti, come nota Schwarze (2015: 2) «sin dalla sua nascita come mezzo di comunicazione di massa nel primo Ottocento la stampa italiana non ha mai cessato di commentare i problemi legati alla questione della lingua».

1. I preziosi contributi di Bonomi (2002), Bonomi/Morgana (2003; 2016), Dardano (1973; 2002), Gatta (2014), Gualdo (2017), Scavuzzo (1988), Castronovo/Ricuperati/Capra (1976), oltre a fornire l'esame linguistico di alcuni quotidiani in varie epoche, offrono una panoramica generale dell'evoluzione del giornalismo dal Settecento ai giorni nostri.

2. L'importanza dei fratelli Verri e di Baretti nel panorama del giornalismo settecentesco è stato recentemente oggetto di due convegni: Giuseppe Baretti, i fratelli Verri, Cesare Beccaria, e i periodici del Settecento. Lingua e storia (Milano, 12-13 dicembre), organizzato presso l'Università degli Studi di Milano da Daniela Marcheschi, Silvia Morgana, Giuseppe Polimeni, Massimo Prada, Sabine Schwarze, Giuseppe Sergio; Giuseppe Baretti scrittore europeo (Torino, 5-6 dicembre 2019), organizzato dall'Accademia delle Scienze di Torino con il patrocinio dell'Università degli Studi di Torino, del Dipartimento di Studi Umanistici dell’Università di Torino e dell'Università Italo-Francese. 
Per familiarizzare con il concetto di «tradizione discorsiva» prendiamo in prestito le parole di Wilhelm (2005: 157):

Chiamiamo tradizione discorsiva l'insieme dei testi, orali o scritti, che vengono prodotti secondo una precisa norma discorsiva [...]. Se parliamo della tradizione discorsiva 'articolo di fondo' ci riferiamo, quindi, all'insieme dei testi prodotti nel tempo secondo questa specifica norma discorsiva, cioè a una serie di testi storicamente determinata. Questo ci fa capire che possiamo fondare una teoria dei tipi di testo solo se prendiamo in considerazione la dimensione storica. Le norme discorsive sono sottomesse a un continuo divenire storico, a un costante cambiamento, vale a dire un continuo riassettarsi e adattarsi ai cambiamenti sociali e culturali nel senso più ampio.

La periodizzazione che proponiamo di seguito si pone sulla scia tracciata da Folena (1986: 194) che, già quarant'anni fa, individuava quattro fasi corrispondenti ai quattro quarti del secolo diciottesimo: il primo periodo era stato denominato età dei «filosofi-critici ed eruditi o dei letterati», seguito dall'età dei «filosofi enciclopedici»; successivamente, si indicava il terzo quarto di secolo come età dei «filosofi-economisti», per poi concludere con l'ultimo quarto caratterizzato dai «filosofi-politici, dei patrioti e dei giacobini». Per semplificare lo spoglio dei periodici, dato che un secolo come il Settecento è caratterizzato da profondi cambiamenti politici e scientifici, abbiamo deciso di raggruppare le quattro fasi storiche di Folena in due metà: la prima, che va dal 1701 al 1750, è contrassegnata dal giornalismo erudito (maggioritario nella prima metà del secolo, cf. Merida 2017), la seconda, che sarà quella su cui ci concentreremo, va dal 1751 al 1796 e si diversifica per la quantità di riviste letterarie, gazzette, fogli e avvisi di stampo economico e politico. Occorre precisare però che i confini tra prima e seconda metà spesso tendono ad allargarsi o a restringersi come nel caso del Nuovo giornale enciclopedico, fondato a Venezia nel 1774 come Giornale enciclopedico per opera di Domenico Caminer e della figlia Elisabetta.

\section{Le note di lingua nei periodici di cronaca}

Senza allargare l'indagine ai periodici più famosi del Settecento, il contributo si propone di individuare una base culturale, ideologica e linguistica ampia e continuativa all'interno dei periodici meno noti. Chiariamo subito che con l'etichetta di periodico a stampa intendiamo quei testi che con una cadenza più o meno regolare vengono stampati e pubblicati nelle varie città d'Italia. Allo stesso modo tratteremo di giornali (quotidiani, settimanali, ecc.) e riviste letterarie, ma con una necessaria premessa che ci permetta di distinguere preliminarmente due fattori: contenuto e cadenza. Le riviste letterarie si occupano quasi esclusivamente di teorie letterarie e scientifiche, senza mai occuparsi di cronaca e politica; hanno, inoltre, una cadenza a lungo termine (mensile, trimestrale, annuale ecc.). I 
giornali, invece, prediligono, a vario titolo, le notizie politiche, economiche e, talvolta, scientifiche; a differenza delle riviste letterarie hanno una cadenza a breve termine (quotidiana, settimanale ecc.) ${ }^{3}$.

La nostra "dimensione storica" di riferimento si avvicina a quella del giornale ottocentesco in cui si assiste a un abbandono della forma elitaria in favore di un giornalismo rivolto a un più ampio pubblico (cf. Masini, 1977: 2-5; Serianni, 1989: 35-36). Tuttavia, l'apice del giornalismo politico si sviluppa in una forbice temporale assai stretta che va dal 1796 al 1799, un triennio denominato dagli storici Triennio repubblicano, nel quale si assiste alla nascita di varie repubbliche dopo le campagne napoleoniche in Italia. Prima di allora le ideologie politiche, che racchiudevano anche una forte ideologia linguistica da parte del giornalista ${ }^{4}$, avevano un loro spazio all'interno delle gazzette, ma senza avere ancora la fisionomia del giornale inteso in senso moderno. Citiamo, solo a titolo d'esempio, due giornali pubblicati con lo scopo di testimoniare le politiche locali: la Gazzetta Patria, già dal secondo numero rinominata Gazzetta toscana (1766-1811), e la Gazzetta urbana veneta (1787-1798), continuatrice del settimanale /l sognatore italiano fondato da Gaspare Gozzi nel 1768. L'azione divulgativa diventava cosi il principale motore dell'editore che avesse voluto stampare un foglio periodico, così come ci dice nell'introduzione del primo numero della Gazzetta Patria Anton Giuseppe Pagani:

E cosa certamente stravagante e vergognosa che nel paese medesimo, dove una volta gli abitatori erano sì diligenti nel raccogliere e trasmettere alla posterità i fatti più memorabili, e quelli ancora che meglio sarebbe stato tacere, sieno al presente cosi trascurati e indolenti che lascino scorrere un secolo per la Toscana cotanto avventuroso senza notarne i più luminosi avvenimenti; di tal maniera che tutta l'istoria patria si riduce in oggi a una semplice gazzetta, la quale non può in verun conto imporre sullo spirito degli uomini, ed acquistare quel credito che volentieri si accorda ad un istorico grave (Gazzetta Patria, 1766: I)

Di là dalla questione meramente ideologica, lo stampatore Pagani offre preziose notizie in merito alla composizione di una gazzetta, non meritevole di essere considerata dagli ambienti intellettuali un foglio contenente «cose frivole e puerili bagattelle» (Gazzetta Patria, 1766: 1) e adatta al grande pubblico per via della sua notevole sveltezza argomentativa data dalla conoscenza dettagliata degli argomenti e della terminologia specialistica:

3. Sono gli stessi giornalisti a darci preziose informazioni relative alla cadenza periodica di un giornale: si veda, per esempio, l'introduzione al Giornale de' letterati d'Italia (1710: 46) in cui si mette in discussione l'appartenenza al genere giornalistico della Galleria di Minerva, pubblicata dal 1696: «Vero è che non è affatto cessata la Galleria di Minerva, la quale cominciò a stamparsi in foglio in Venezia nel 1696. Ma non può questa tener luogo di giornale, così perché non si dà regolarmente di mese in mese, onde in 14 anni appena compiè il sesto tomo».

4. Sul rapporto tra ideologia politica e ideologia linguistica si rinvia alla seconda parte di questo contributo, che tratterà specificatamente dei periodici del periodo giacobino (1796-1799). 
Le Gazzette [...] dagl'ingegni però cari a Minerva son tenute per la specie di composizioni la più dura, e la più utile al pubblico, che sia comparsa in abbigliamento di serva ed accorda a' componitori delle medesime quella stima che meritano; mentre elleno esigono perizia dei linguaggi e di tutti i loro termini: facilità e franchezza di scrivere; una precisione di dettaglio per narrare le cose nettamente, senza superfluità di parole (Gazzetta Patria, 1766: 1).

Pagani continua il suo elenco di elementi utili per la composizione della gazzetta proponendo alcune considerazioni in merito alla conoscenza dello scrittore la cui bravura risiede nell' "esser abile a parlare di guerra; essere appieno informato di tutto quello che riguarda la geografia, l'istoria del tempo e quella delle famiglie più luminose» (ib.). Le parole di Pagani rivelano un fatto finora rimasto in ombra e utile per la costruzione del pensiero settecentesco in merito alla circolazione delle gazzette: in particolare, si nota che una parte di intellettuali considerava tali periodici a stampa semplici notiziari senza alcun pregio. È noto, e lo stesso Pagani lo scrive più volte, che al compilatore di un foglio periodico era necessaria una cultura ampia e la padronanza di un lessico dettagliato.

L'interesse ad allargare la platea di lettori proviene anche da un altro periodico, il Magazzino italiano, stampato a Livorno nel 1752 per i tipi di Antonio Santini. Sul modello dei magazines inglesi (Gremigni, 2008: 164), il Magazzino italiano si fece portavoce, pur per brevissimo tempo, delle tendenze culturali italiane ed europee. Anche in questo caso è di notevole importanza l'azione divulgativa dei redattori, che dichiarano di scrivere per il pubblico e non «per i dotti» (Magazzino italiano, 1752: 3). Un pubblico che non ha avuto la possibilità di approfondire gli studi nelle scienze e che, di norma, opera in altri settori, non appartenenti al sapere tecnico-scientifico. L'introduzione alla rivista è particolarmente vivace e mostra gli intenti che mossero gli autori alla fondazione e pubblicazione del Magazzino italiano:

Noi scriviamo per quelle persone che distratte da impieghi o da cure più utili alla società non posson consacrare che una piccola parte del tempo alla lettura. Scriviamo per quelli che non hanno potuto darsi alle scienze, che si lamentano di non possederle, e che ne sospirano qualche notizia. Per questi servirà mirabilmente la nostra opera, quale se non fosse altro darà loro la definizione di molte cose, che prima di leggerla potrebbero spaventarli (Magazzino italiano, 1752: 3).

Pur mantenendo una configurazione di «un centone di notizie ufficiali, feste, cerimonie, partenze e arrivi di personaggi illustri, di spettacoli teatrali» (Bonomi, 2010: online), le gazzette permettono di leggere notizie preziose relative ad alcune indicazioni pratiche da parte degli organi politici di varie città o delle accademie culturali. A differenza dei periodici politici giacobini, quelli pubblicati tra gli anni '50 e '90 del Settecento sono privi di giudizi ideologici dei redattori e offrono quasi esclusivamente la semplice notizia di cronaca. Un caso particolare è offerto dal seguente articolo: «Si ordina ai medici, riguardo ai medicamenti da somministrarsi agli ammalati, di scrivere le ricette intieramente distese in idioma italiano, col nome del fisico in carattere chiaro, e non colla cifra come si praticava in passato» (Notizie del mondo, 97: 16 dicembre 1786), in cui si informa il lettore di un'importante novità 
riguardante la decisione del governo degli Asburgo d'Austria in merito alla concessione all'Istituto Pio di Santa Corona di Milano di poter scrivere in italiano le ricette mediche: un elemento degno di nota che ci consente di mettere in evidenza la scelta editoriale del giornale.

Per cogliere gli interventi dei redattori delle Notizie del mondo occorre guardare le note di commento, puntualmente segnalate da un asterisco e inserite a piè di pagina. L'importanza del commento redazionale è evidente quando in una traduzione di un articolo politico si giustifica la voce arresto, dal francese arrêt 'decisione di un tribunale o di un organo legislativo; sentenza, decreto' (cf. GDLI: S.v. arresto) $)^{5}$ :

Il vocabolo Arresto, in significato di Decisione, di Sentenza, di Pronunzia, è toscanissimo, come quello ch'è riportato in tal significato non solo dall'Antonini, dall'Abate Alberti, dagli altri Dizionarj, ma eziandio dalla Crusca, la quale, sotto la voce di cui si tratta, dice precisamente Arresto in alcun luogo usasi per lo stesso che Sentenza, Decreto. Si usa poscia in questi foglj per esprimersi le Risoluzioni del Parlamento, a fine di distinguersi queste Risoluzioni d'una Corte Suprema non sottoposte ad alcun appello, così dalle Sentenze dalle Corti subalterne soggette all'appello a Tribunali Superiori ed in ultima istanza al Parlamento medesimo, come dai Decreti del Consiglio in cui risede l'autorità reale. (Notizie del mondo, 75: mercoledì 19 settembre 1787).

Il ricorso ad alcuni dei più importanti vocabolari settecenteschi per spiegare una parola che a quell'altezza cronologica era già entrata nell'uso da circa un secolo (la voce arresto entra già nella Ill edizione della Crusca) e la definizione di «vocabolo [...] toscanissimo» somigliano a una difesa preliminare alle possibili critiche che l'uso del francesismo avrebbe potuto provocare.

Anche le novità bibliografiche offrono ai compilatori un'occasione per ragionare sull'importanza di alcune traduzioni italiane di opere straniere. Il fatto che le traduzioni di opere tecnico-specialistiche richiamino l'attenzione degli studiosi è già evidente nel primo Settecento in riviste come il Giornale de' letterati d'Italia, che sottolineava l'importanza di accrescere il lessico scientifico italiano attraverso le traduzioni: un'eredità culturale ben accolta anche dalle Notizie del mondo. All'indomani della pubblicazione della Biblioteca di Gius nautico, stampata a Firenze nel 1785, un redattore delle Notizie del mondo descriveva cosi l'opera:

5. Il passo in questione è il seguente: «Questi sono i principi che il Parlamento, attualmente in Troyes, vuole applicarsi a sviluppare. II medesimo, in continuazione dell'Arresto del di 27 dello scorso agosto, ne fece un altro, nel 28, contro le Lettere di Sigillo». (Notizie del mondo, 75: mercoledì 19 settembre 1787). 
Era desiderata in Italia una copiosa raccolta di leggi e di moderni trattati delle più culte nazioni riguardanti il commercio e le questioni marittime. [...] All'opera finalmente darà termine un vocabolario, in cui saranno spiegati con la possibile esattezza tutti i termini di commercio e di nautica, non solo in lingua italiana, ma ancora in lingua francese, inglese, olandese e spagnuola. È manifesta l'utilità di un si fatto vocabolario; perocché per mezzo di esso potranno evitarsi le molte questioni che nascono dagli equivoci e sbagli enormi presi spesso dai traduttori intorno al vero significato de' termini rozzi ed antiquati, che formano un linguaggio particolare delle genti di mare nei consolati, contratti polizze ecc. (Notizie del mondo, 21: sabato 12 marzo 1785).

L'importanza di un trattato che contenesse al suo interno un vocabolario formato da un lessico tecnico è, per il redattore, la notizia più importante da offrire al pubblico.

\section{Gli interessi linguistici del giornalismo erudito}

A proposito dei giornali veneti del Settecento, Folena (1986: 200) confermava quanto fosse rapida «la circolazione e l'assimilazione di idee e di parole nuove». Tale affermazione può essere estesa, più genericamente, alla situazione dei giornali italiani. In particolar modo, le pagine delle riviste letterarie descrivono due fatti importanti: 1) una circolazione del sapere scientifico ampia e continuativa; e in questo panorama ricorrono quasi sempre gli stessi nomi: Galileo, Magalotti, Zendrini, Vallisneri, Redi; 2) la lingua in cui codificare tale sapere. Senza dover scavare a fondo, sfogliando le pagine del Giornale d'Italia (1764-1797) è possibile leggere alcuni articoli interessanti per la ricostruzione del pensiero linguistico e scientifico settecentesco. Specifichiamo subito che spesso le opinioni linguistiche dei giornalisti provengono dalle recensioni di libri. Così un redattore nel decimo numero del 1766 del Giornale d'Italia, in una recensione alla Meccanica sublime di Domenico Bartaloni, informava vivacemente i lettori intorno alla possibilità, da parte degli scrittori italiani, di scrivere di meccanica, geometria o algebra in italiano, notando come molti scienziati italiani non fossero in confidenza con i trattati in latino?.

Sempre nella prima metà del Settecento si cominciano a stampare diverse riviste esclusivamente rivolte alle cosiddette novelle letterarie. Le informazioni date dai giornalisti sono molto chiare e già nell'introduzione al Giornale de' letterati d'Italia (1710: 13) si annunciano metodi e finalità dei periodici eruditi, definiti opere «che regolatamente di tempo in tempo ragguaglio danno de' varj libri, ch'e-

6. Il Giornale d'Italia spettante alla scienza naturale e principalmente alle arti e al commercio fu un settimanale di otto pagine legato alle accademie agrarie venete.

7. Giornale d'Italia, n. X, 1766, p. 76: «ll dotto autore dice nella prefazione d'aver trattato della meccanica sublime in lingua italiana, perché la nostra lingua non manca d’ogni espressione per qualunque arte o scienza; e di più non si fa vedere, perché, conforme riflette il Viviani, addomesticar non debbasi per queste dottrine la nobilissima favella nostra, avvalorati maggiormente al suo esempio e da quello degli altri autori sommi [...]. Io aggiungerei che conosco de' meccanici, de' geometri e degli algebristi stimabili, tutti italiani, o dimoranti in Italia, i quali non sono addomesticati col latino; onde per loro sarebbe anche bene di tradurre i trattati matematici latini in lingua nostra volgare». 
scono di nuovo in luce». Le stesse finalità arrivano anche in alcune riviste fondate nella prima metà e protratte fino alla seconda metà del Settecento: le Novelle della repubblica delle lettere pubblicate da Angelo Calogerà, pubblicate fra il 1729 e il 1776, e le Novelle letterarie pubblicate in Firenze curate da Giovanni Lami dal 1740 al 1769, per poi continuare fino al 1791 sotto altri due diversi direttori. Com'è già stato notato (cf. Castori, 2010: 73-80; Merida 2017: 17-18), il periodico fondato da Lami si configura come un importante strumento ideologico per due motivi: da una parte segue l'impostazione dei giornali veneziani precedenti; dall'altra vanta una forte tradizione linguistica e culturale. L'elogio della fiorentinità riecheggia già nella prefazione al primo numero della rivista in cui, oltre a elencare i migliori ingegni che Firenze produsse fino a quell'epoca, si tessono le lodi delle accademie; di particolare interesse il commento all'Accademia della Crusca: «la celebratissima Accademia della Crusca, non mai bastevolmente lodata, e sempre temerariamente ed inutilmente assalita» (Novelle letterarie, 1740: VIII). Le novità bibliografiche del Lami, corredate da veri e propri giudizi critici, riportano costantemente notizie relative alle attività della Crusca e dei suoi accademici. Ampio spazio è dedicato alla pubblicazione del Compendio del Vocabolario degli Accademici della Crusca da parte Domenico Maria Manni nel 1739. La posizione ideologica di Lami nei confronti di quest'opera è abbastanza evidente se messa a confronto con la segnalazione che nello stesso anno, il 1740, compare nel Giornale de' letterati d'Italia. Nelle parole di Lami, a differenza di quelle del Giornale de'letterati, si intravede un'operazione di promozione data dal fatto che l'Accademia della Crusca ha sede a Firenze: ecco che, per le Novelle letterarie, la quarta impressione del Vocabolario è considerata «magnifica» e che l'opera del Manni è considerata «scrupolosa fatica»; nella frase di chiusura, infine, è racchiusa l'azione propagandistica delle Novelle letterarie8.

Qualche anno dopo la fine della direzione di Lami delle Novelle letterarie, uscivano le Efemeridi letterarie, una rivista erudita stampata a Roma dal 1772 al 1798. L'impostazione del periodico è simile a tutte le altre riviste, ma la densità e la qualità delle segnalazioni bibliografiche al suo interno rivelano ampi interessi linguistici. Ancora una volta il quadro è occupato dal recensore, che sfoggia il proprio punto di vista ogni qual volta l'occasione lo permetta. Esemplare, quanto alle commedie, il comportamento nei confronti delle opere scritte in italiano da Goldoni. Lo spunto per parlare di lingua è offerto dalla notizia della premiazione di due commedie teatrali da parte della Reale Accademica

8. Giornale de' letterati d'Italia (1740: 440): «È già uscito il Compendio del Vocabolario della Crusca formato sull'edizione quarta del medesimo, e stampato dal Sig. Domenico Maria Manni in Tomi V. Nella prefazione assicura l'Autore di questo Compendio di non aver omesso dizione alcuna di quelle che si portano nell'ultima edizione del Vocabolari»; Novelle letterarie pubblicate in Firenze (1740: 148-150): «Essendo restata felicemente terminata nell'anno scorso la magnifica quarta edizione del gran Vocabolario degli Accademici della Crusca; e considerando il Sig. Domenico Maria Manni, che quest'opera da esso con somma esattezza ed eleganza stampata essendo riuscita molto voluminosa, e dispendiosa, non poteva esser utile sennon ad alcuni generi di persone [...]. Ė stato altresì animato a tale impresa dall'osservare che utilità grande era avvenuta agli Studiosi dal Compendio dell'Edizione terza del medesimo Vocabolario, pubblicato da un illustre Letterato Veneto, e con sommo applauso ricevuto, di modo tale che fu duopo farne, alle universali richieste, molte ristampe. Abbenché siasi affaticato il Sig. Manni di far questo suo Compendio più breve che fosse possibile; nientedimeno egli è stato necessario di allargarsi in cinque tomi in quarto, perché non ha voluto omettere dizione nessuna [...]. Quantunque la grand'opera del Vocabolario abbia sopra questa del Compendio i suoi particolari pregi a lei sola riservati; tuttavia la perizia del Sig. Manni in questo genere di sapere ben nota al Mondo Letterario, e la gran diligenza, e scrupolosa fatica impiegata nel far questo Compendio, ci fanno sperare, che egli sarà ricevuto con applauso universale». 
Deputazione di Parma, il Prigioniero di Francesco Albergati Copacelli (vincitore del primo premio nel concorso del 1773) e la Marcia dell'abate Francesco Marucchi (vincitore del secondo premio nello stesso concorso). Il giornalista, in due recensioni distinte, tesse le lodi del primo autore e lamenta l'assegnazione del secondo posto attribuito a Marucchi, sostenendo che l'opera in questione «sia stata onorata della seconda corona unicamente per animare la gioventù e per la scarsezza di concorrenti» (Efemeridi letterarie, 1773: 285). La critica, che si rivela abbastanza aspra nei confronti del modo con cui si tratta l'intreccio, spende una parola positiva nei confronti dello stile «migliore di quello che usa il Goldoni» (Efemeridi letterarie, 1773: 285). Contrariamente alle critiche di ascendenza gozziana, l'effemeridista elogia la maestria con cui Goldoni colora di umanità le scene, ma non ne apprezza (quando è presente) l'uso dell'italiano al posto del veneziano:

A proposito del Goldoni, oh se quest'uomo studiata avesse davvero la lingua italiana e un poco più nobilitate le idee! [...] Aggiungeremo una osservazione sopra l'illustre Sig. Goldoni, poeta che noi pregiamo assaissimo: quando egli scrive nel suo natio dialetto veneziano, è purissimo, originale, e si legge dagl'intendenti con infinito piacere, ma guai allora ch'egli pretende di scrivere in italiano! La nostra riflessione è tanto vera che il Cerloni stesso, autore, se altri mai, di stravaganti commedie [...], ove scrive ancor'esso nel suo dialetto napoletano, diletta infinitamente, dipinge gli originali al naturale. (Efemeridi letterarie, 1773: 285).

Per accrescere il valore dialettale dell'opera goldoniana, il recensore fa un richiamo anche all'autore napoletano Francesco Cerlone ${ }^{9}$.

Com'è ben noto, il rinnovamento linguistico e culturale che stava avvenendo in Italia durante il Settecento (cf. Folena, 1983: 7-10; Matarrese, 1993, 41; Serianni, 1998: 187) portò con sé un ventaglio di innovazioni lessicali e sintattiche non estranee agli occhi dei giornalisti. Nel numero XXXIX delle Efemeridi del 1774 una recensione al Saggio filosofico sul matrimonio di Melchiorre Delfico rivela la posizione del periodico nei confronti di alcuni usi lessicali (cf. Dardi 1992: 296):

In fine non meneremo mai buono all'Autore che faccia il torto alla nostra Italia di crederla così scarsa e meschina d'espressioni, che abbia a prenderne imprestito non poche dalla Francia, come regrettare, disabusare etc. Ciò non ostante siam sicuri che la sua opera incontrerà gradimento. Noi certamente ci dichiariamo non essere schiavi delle severe leggi della crusca, la quale non ammette che le parole bagnate nell'Arno, ed autorizzate da fra Guitone o dal Passavanti, ma non approviamo che se ne adoprino delle nuove quando abbiamo le loro corrispondenti nella nostra lingua, e talvolta più eleganti di quelle, che affettatamente andiamo ad accattare dai nostri avversari. (Efemeridi letterarie, 1774:311-312).

9. Il paragone tra Cerlone e Goldoni non è usuale. Cerlone, infatti, non ebbe un buon riconoscimento per le traduzioni napoletane dell'autore veneziano che anzi descrisse così la sua opera versificatoria: «Se sei ricamator come poeta / poveri drappi e sventurata seta». Cf. Giovanardi (1979). 
Siamo su un piano ben diverso rispetto alle vivaci parole che si possono trovare nei periodici a stampa posteriori di qualche anno. In questo caso, anche se non si assiste a una demonizzazione nei confronti dei francesismi usati da Delfico, viene dichiarata una preferenza verso le corrispettive parole italiane definite «più eleganti»» ${ }^{10}$. Un giudizio, quello dell'eleganza e della bellezza della parola, che vive durante tutto il Settecento e continua fino ai giorni nostri, ma con una ovvia differenza: chi scrive la recensione non disapprova tutti i prestiti, ma soltanto quelli non necessari alla lingua italiana. Si aggiunga, inoltre, che l'apertura linguistica di un periodico a stampa settecentesco contrario alle imposizioni dell'Accademia della Crusca si inserisce nel solco della «lingua comune» individuata da Folena (1983: 7-10).

Ben altra messe si potrebbe raccogliere guardando a due recensioni su uno stesso tema pubblicate su due diverse riviste: le Novelle letterarie e le Efemeridi. Nelle Novelle letterarie del 27 ottobre 1780 vengono riportati alcuni versi dell'«anonimo traduttore» seguiti da un breve commento linguistico (corsivo nel testo):

Il verso ha dell'armonia e lo stile avrebbe dell'eleganza se non fosse asperso di qualche negligenza grammaticale, come possin per possano (p. 8), testimonio invece di testimone (p. 35), voi vorresti in cambio di voi vorreste (p. 52). Non approveranno neppure i lettori di gusto purgato che si adopri il mai in senso negativo senza il non (p. 11). Madama invece dell'italiano vocabolo Donna, o Signora (p. 12) ed altre simili disattenzioni che non tolgon però il pregio sostanziale della traduzione. (Novelle letterarie, 1780: 675).

Gli esempi indicati dal recensore vengono puntualmente ripresi e giustificati dall'articolo apparso qualche mese dopo nelle Efemeridi letterarie (precisamente nel numero XIII del 31 marzo 1781): una prova di quanto ampia fosse la diffusione dei giornali nel territorio extraregionale. II tono polemico, rivolto all'articolo delle Novelle letterarie, si rivela nella seconda metà della recensione, dopo aver esteso il nome del traduttore, cioè il canonico Orazio Maccari. In generale il redattore si mostra sensibile all'eterogeneità linguistica che emerge dalla traduzione dei versi. Non c'è nessuna sorpresa nell'uso del mai usato negativamente senza il non; né fa specie l'occorrenza di testimonio al posto di testimone. Sono notevoli, semmai, le rapide incursioni storico-linguistiche del giornalista, il cui impegno è dimostrare l'uso letterario di alcune forme considerate scorrette: si passa dalle citazioni di Machiavelli a quelle di Dante, Boccaccio o Ariosto anche per giustificare il sostantivo madama in luogo di donna. L'apertura nei confronti di madama e di altri fenomeni dà prova di una coscienza

10. Un appunto sulla voce disabusare, prestito dal francese désabuser. La prima attestazione, secondo il GDLI (s.v.), è datata 1669 nella Vita di Sisto V di Gregorio Leti: «Credeva che non vi fosse differenza tra l'uno e l'altro de' Cardinali, havendo già inteso ch'erano insieme fratelli e per ciò s'era imaginato che tra di loro si dividevano la rendita della Chiesa, e volse per disabusarsi esser meglio informato» (Leti, 1669: 292). A distanza di un secolo, secondo gli autori delle Efemeridi, il termine disabusare rientra ancora tra le parole da non usare e viene messo sullo stesso piano di regrettare, voce nuova che fa la sua prima apparizione in un testo di Lione Pascoli, databile al 1741 (cf. GDLI, s.v.). Sul verbo disabusare si leggano le parole di Dardi (1982: 111-112): «Calco infrequente nei secoli XVII e XVIII [...], tuttavia deve aver avuto un qualche corso, se lo troviamo satireggiato dal Maffei [...] e, più di un secolo dopo, biasimato dai puristi». 
linguistica maturata grazie a una conoscenza diretta delle fonti letterarie e veicolata anche dall'uso del Vocabolario della Crusca:

Fracassi, precipizj perché nell'Irene si adopera la parola Madama. Ma perché non fanno fracasso col loro dizionario della crusca, il quale dice che cosi si chiamano le donne di grand'affare, e che cosi l'hanno usata il Boccaccio, l'Ariosto ed altri infiniti? La voce certamente è d'origine francese, ma è divenuta anche essa nostra nazionale, com'è divenuta italiana la voce Monsignore da che tornò la corte romana da Avignone. Guai a noi poveri Efemeridisti romani, se i Signori Novellisti fiorentini ci pesassero con questa loro delicata bilancia, perché malgrado i decreti d'Apollo, i nostri stampatori si direbbe che vogliono accostumare assolutamente gli orecchi di S.M. a peggiori eleganze, nelle quali non è certamente capace di cadere il Sig. canonico Maccari. (Efemeridi letterarie, 1781: 103).

All'Accademia della Crusca rimandano anche alcune pagine del Nuovo giornale de' letterati d'Italia (1773-1790) diretto da Girolamo Tiraboschi ${ }^{11}$. Di particolare interesse la decisione editoriale di pubblicare in cinque puntate, dal 1787 al 1789, la prima edizione delle Lettere di Diodoro Delfico a Lesbia Cidonia sopra gli epigrammi del gesuita mantovano Saverio Bettinelli (cf. Dillon Wanke, 1998: 125-147). La pubblicazione di un'opera letteraria all'interno di una rivista, pur essendo una pratica comune, specie durante un secolo come il Settecento caratterizzato dalla forte presenza di riviste letterarie, denota un intento non solo divulgativo ma anche ideologico (cf. Gallo 2017: 499). Le Lettere di Bettinelli, infatti, non sono prive di riferimenti al contesto culturale dell'epoca: per esempio, la Lettera X, pubblicata nel tomo XXXVIII nel 1787, si intrattiene sullo «spinaio grammaticale della differenza tra i linguaggi» (Nuovo giornale de' letterati d'Italia, 1787: 19). Nel condurre l'argomento, Bettinelli fa riferimento anche all'altalenante fortuna dell'Accademia della Crusca, affermando che in quel periodo «sembra nascere un'anarchia su le rovine dell'Accademia della Crusca e sul fondamento della filosofia dominante, la quale sdegna l'antiche leggi in ogni cosa anche più grave». La lettera continua con il confronto fra italiano e francese: seguendo il dibattito linguistico contemporaneo, Bettinelli fa cenno all'ordo verborum e ai possibili problemi che scaturiscono dall'inversione. Insomma, è importante non tanto l'intero contenuto del testo, quanto la sua presenza all'interno di un periodico a stampa che esibisce «tutta l'attenzione e tutta l'acutezza del Tiraboschi al riguardo delle tendenze più avanzate della letteratura contemporanea» (Mari, 2013: 66).

Entro il recinto della teoria linguistica può rientrare l'atteggiamento di Tiraboschi quando indossa i panni di apologeta della lingua italiana nell'articolo relativo alla traduzione dell'/liade di Cesarotti. È questo uno dei casi in cui si assiste a un dibattito teorico traduttologico in cui l'ideologia linguistica del giornale è chiara. La risposta di Tiraboschi all'intervento dello scrittore spagnolo Esteban Arteaga

11. Sulla preparazione alla pubblicazione delle Lettere di Diodoro Delfico a Lesbia Cidonia si veda il carteggio TiraboschiBettinelli riportato da Mari (1988: 228-279). Inoltre, Dillon Wanke (1998: 133) riporta un'informazione importante sulla ricostruzione dell'opera di Bettinelli: «La lettera del 18 novembre 1878 [...] ci lascia credere che Bettinelli non fosse pienamente soddisfatto dell'edizione tiraboschiana». Più in generale, sulle lettere di Bettinelli cf. Forner (2019) e Cappelletti (2019). 
è caratterizzata da toni pacati e da una lunga ed esauriente analisi rivolta alla difesa della lingua italiana:

Non è qui nostra intenzione di fare l'Apologia della nostra lingua, e di difenderla dalle taccie, che l’Ab. Arteaga le appone, né di rammentare alcune traduzioni che abbiamo degli Autori greci e latini, le quali a noi sembran molto pregevoli. Ma se la difficoltà anzi l'impossibilità di tradurli adeguatamente mostra la povertà della lingua, ci dica egli di grazia, qual sia la lingua d’Europa che possa dirsi abbondante e copiosa. [...] La ragione che rende sì rare e sì difficili le buone versioni, deesi ripetere, secondo la nostra opinione, dalla somma difficoltà di ben conoscere il genio e l'indole delle due lingue da cui e in cui si traduce, e dal saper adattare le espressioni dell'una a quelle dell'altra, in modo che il sentimento, l'affetto, l'immagine, l'armonia dell'originale nulla venga a soffrirne. (Nuovo giornale de' letterati d'Italia, 1787: 93).

Per spiegare le critiche di Arteaga alla lingua italiana è necessario far presente che la traduzione di Cesarotti si inserisce nel secolare dibattito della traduzione introdotta già a inizio secolo da Maffei e portata avanti da personalità come Torelli e Pompei. D'altro canto, la libera interpretazione dell'/liade rompe con la tradizione di traduzioni fedeli all'originale (cf. Mari, 1990: 1-75); lo stesso Tiraboschi, mediante il concetto del "genio e l'indole delle due lingue", sostiene la scelta di Cesarotti, che l'anno precedente, nel «Ragionamento preliminare storico-critico» all'/liade, aveva affrontato la questione della traduzione dei classici (cf. Battaglia, 1971:431). La polemica, tutta svolta nell'arco di pochi anni, vedrà anche una successiva testimonianza di Cesarotti nella traduzione dell'/liade pubblicata nel 1795, nella quale si fa riferimento alle critiche mosse durante gli anni precedenti dagli «Omerolatri e [...] Fidelisti» (Cesarotti, 1795: IX) (cf. Schwarze, 2020). La recensione dell'lliade, inoltre, offre a Tiraboschi l'opportunità di inquadrare le innovazioni stilistiche di Cesarotti all'interno del canone proposto dalle varie edizioni del Vocabolario della Crusca, rispondendo, passo dopo passo, alla polemica mossa da Arteaga ${ }^{12}$, che definiva «adeguata» la traduzione cesarottiana di Ossian per via della sua rottura con i «ceppi dell'autorità»:

Qual è questa autorità, i cui ceppi ha infranti l'Ab. Cesarotti? Sembra ch'ei voglia intendere qui dell'Accademia della Crusca, che da alcuni, e da lui stesso ci si rappresenta come dichiarata nimica di ogni novità nella lingua italiana. Ma in ciò ancora ei s'inganna di molto. E basti il riflettere che in ogni nuova edizione del Vocabolario si sono aggiunte parole ed espressioni che alle precedenti mancavano, e che da autori recenti erano state introdotte. Né è questo privilegio accordato a' soli toscani, ma l'Accademia ne è stata cortese anche agli stranieri. Così ella ammise già tra scrittori che facean testo di lingua, l'Ariosto, il Bembo, il Tasso, il Caro, il Guarini, il Castiglione; così più recentemente adottò il P. Segneri, e le nuove voci da essi introdotte, ricevette non altrimenti che sue. Se dunque l'Ab. Cesarotti ha creato nuove attitudini nello stile, ha usato di un diritto, che la ragione e l'autorità gli accordavano. E questo

12. Pochi anni prima, nel 1784, Arteaga lamentava la crisi della prosa, dovuta alla mancanza di un modello unitario per la letteratura (cf. Serianni, 2012: 108). 
anzi torna a nuova gloria della lingua italiana, e ne fa conoscere l'abbondanza, perciocché egli usando pure di questa lingua e non creandone una nuova, e servendosi comunemente di parole e di espressioni in essa già approvate, un nuovo stile ha saputo in essa introdurre. (Nuovo giornale de' letterati d'Italia, 1787: 94-95).

L'articolo appena citato si mostra, da un lato, particolarmente precoce nel presentare alcune tendenze linguistico-letterarie dell'epoca (nella fattispecie, la difesa di un nuovo modo di tradurre i classici greci e latini), come per esempio la traduzione dall'inglese dei Canti di Ossian, tradotti da Cesarotti con un nuovo metodo, in grado di «far parlare i personaggi dell'Ossian come avrebber parlato se conosciuta avessero la nostra lingua» (Nuovo giornale de' letterati d'Italia, 1787: 96), oltreché l'influenza che da lì a poco avrebbe avuto anche sulle traduzioni dalle lingue classiche ${ }^{13}$; dall'altro dimostra, ancora una volta, l'importanza dell'Accademia della Crusca, ancora tacciata di essere «nimica di ogni novità nella lingua italiana». Resta notevole che a movimentare (e ad inserirsi) nel dibattito linguistico e culturale dell'epoca siano periodici a stampa, continuamente esposti alle novità del Settecento e iniziatori di un processo divulgativo che fungerà da base per la stampa ottocentesca.

\section{Prime conclusioni}

Gli articoli qui presentati sono la testimonianza dell'attenzione dei periodici a stampa al naturale processo evolutivo della lingua e ciò che più colpisce è la tendenza a inserire le discussioni linguistiche anche al di fuori degli articoli di critica letteraria. Come si è visto, si tratta di commenti linguistici anche minimi che rivelano tuttavia l'eccezionale capacità divulgatrice della stampa, rivolta anche a chi non poteva entrare a far parte del dibattito intellettuale dell'epoca. D'altronde quasi tutte le riviste letterarie e i giornali sono chiari su questo punto: riportare notizie letterarie e tradurre dai giornali esteri per offrire a chiunque la possibilità di entrare in contatto con la realtà contemporanea. In linea con gli articoli presenti nella stampa di primo Settecento (cf. Merida 2017), quelli qui analizzati aggiungono un ulteriore tassello necessario alla ricostruzione di un periodo storico-culturale in cui la questione della lingua era l'argomento principale per tutta la stampa europea.

Nei periodici presi in esame si intravedono già le prime caratteristiche dei giornali ottocenteschi, che mischiano politica e letteratura anche attraverso il commento linguistico (si veda per esempio il giornale diretto da Elisabetta Caminer); tuttavia, la loro configurazione condivide ancora i caratteri del giornalismo erudito del Settecento. Come si dimostrerà in una seconda parte di questa ricerca, interamente dedicata alla stampa giacobina, bisognerà attendere l'arrivo di Napoleone in Italia, e la conseguente diffusione dei giornali, per vedere quanto l'ideologia politica e l'ideologia linguistica siano al centro del dibattito giornalistico.

13. Sulla traduzione di Cesarotti dei Canti di Ossian si vedano almeno i recenti lavori di Bianco (2016: 10-28, 2018: 261292) e Roggia (2013: 109-220). 


\section{Bibliografia}

Battaglia, Salvatore (1971), «La critica classicista di Vincenzo Gravina», Filologia e Letteratura, n 17 , p. 427-449.

Beccaria, Gian Luigi (1973), «ll linguaggio giornalistico», in Gian Luigi Beccaria (ed.), I linguaggi settoriali in Italia, Milano, Bompiani, p. 61-89.

Bianco, Francesca (2016), «Traduzioni a confronto: "I canti di Ossian” di Le Tourneur e Cesarotti», Testo, n 71 , p. 10-28

Bianco, Francesca (2018), «Le poesie di Ossian. Osservazioni critiche di Cesarotti a Le Tourneur», Critica letteraria, vol. 179, n² 2, p. 261-292.

Bonomi, Ilaria (2002), L'italiano giornalistico. Dall'inizio del Novecento ai quotidiani online, Firenze, Cesati.

Bonomi, Ilaria (2010), Lingua dei giornali, in Enciclopedia dell'italiano, disponibile su http://www. treccani.it/enciclopedia/lingua-dei-giornali_(Enciclopedia-dell'Italiano)/. [Sito consultato il 15 giugno 2020]

Bonomi, Ilaria, Stefania De Stefanis Ciccone e Andrea Masini (ed.) (1990), /l lessico della stampa periodica milanese, Firenze, La Nuova Italia.

Bonomi, Ilaria e Silvia Morgana (2003), La lingua italiana e i mass media, Roma, Carocci.

Bonomi, Ilaria e Silvia Morgana (2016), La lingua italiana e i mass media, nuova edizione, Roma, Carocci.

Cappelletti, Cristina (2019), «Saverio Bettinelli tra libri di lettere e “romanzi”», Testo, n 77, p. 119-134.

Castori, Loredana (2010), «Le novelle letterarie dei giornali del Settecento», Misure critiche. Nuova serie, a. $9, n^{\circ} 1$, p. 64-85.

Castronovo, Valerio, Massimo Ricuperati e Carlo Capra (ed.) (1976), La stampa italiana dal Cinquecento all’Ottocento, Roma-Bari, Laterza.

Cesarotti, Melchiorre (1786), «Ragionamento preliminare storico-critico», in L'lliade d'Omero recata poeticamente in verso sciolto italiano insieme col Volgarizzamento letterale del testo in prosa, vol. 1, Padova, Brandolese, p. 1-235.

Cesarotti, Melchiorre (1795), «Avvertimento preliminare», in L'lliade o la morte di Ettore. Poema omerico ridotto in verso italiano, Venezia, Tipografia Pepoliana presso Antonio Curti, p. V-XI.

Dardano, Maurizio (1973), Il linguaggio dei giornali italiani, Bari-Roma, Laterza.

Dardano Maurizio (2002), «La lingua dei media», in Valerio Castronovo e Nicola Tranfaglia (ed.), La stampa italiana nell'età delle TV. Dagli anni Settanta a oggi, Roma-Bari, Laterza, p. 243-285. 
Dardi, Andrea (1982), "L'influsso del francese sull'italiano tra il 1650 e il 1715 (IX). Calchi formali», Lingua nostra, vol. XLIII, nº 4, p. 107-116.

Dardi, Andrea (1992), Dalla provincia all'Europa: l'influsso del francese sull'italiano tra il 1650 e il 1715 , Firenze, Le Lettere.

De Mauro, Tullio (1976), «Giornalismo e storia linguistica dell'Italia unita», in Valerio Castronovo e Nicola Tranfaglia (ed.), La stampa italiana del neocapitalismo, Roma-Bari, Laterza, p. 455-505.

De Stefanis Ciccone, Stefania (1971), La questione della lingua nei periodici letterari del primo Ottocento, Firenze, Olschki.

Di Brazzà, Fabiana (2012), "Apostolo Zeno dalla "Galleria di Minerva" al "Giornale de' Letterati"» in Enza del Tedesco (ed.), Il "Giornale de' letterati d'Italia» trecento anni dopo. Scienza, storia, arte, identità (1710-2010), Atti del convegno, Padova, Venezia, Verona 17-19 novembre 2010, PisaRoma, Fabrizio Serra, p. 155-164.

Dillon Wanke, Matilde (1998), «"Bagatellando”. Le lettere di Diodoro Delfico a Lesbia Cidonia sopra gli epigrammi», in Ilaria Crotti e Ricciarda Ricorda (ed.), Saverio Bettinelli. Un gesuita alla scuola del mondo, Atti del Convegno (Venezia, 5-6 febbraio 1997), Roma, Bulzoni, p. 125-147.

Folena, Gianfranco (1983), L'italiano in Europa. Esperienze linguistiche del Settecento, Torino, Einaudi.

Folena, Gianfranco (1986), «"Alla vigilia della rivoluzione francese”. L'italiano due secoli fa tra riforme e rivoluzioni», Lettere italiane, vol. 38, n², p. 193-216.

Forner, Fabio (2019), «Bettinelli scrittore di lettere: precettistica, prassi e letteratura», Testo, n 77, p. 105-117.

Gallo, Valentina (2017), «La critica letteraria in forma epistolare», in Fabio Forner, Valentina Gallo, Sabine Schwarze e Corrado Viola (ed.), Le carte false. Epistolarità fittizia nel Settecento italiano, Roma, Edizioni di Storia e Letteratura, p. 491-500.

Gatta, Francesca (2014), «Giornalismo», in Giuseppe Antonelli, Matteo Motolese e Lorenzo Tomasin (ed.), Storia dell'italiano scritto, vol. 3, Roma, Carocci, p. 293-347.

Giovanardi, Stefano (1979), «Cerlone Francesco», in Dizionario Biografico degli Italiani, Roma, Istituto dell'Enciclopedia Italiana, vol. XXIII, p. 765-768.

Gremigni, Elena (2008), «La stampa periodica a Livorno nel secolo dei Lumi», in Silvia Capecchi (ed.), Giornali del Settecento fra Granducato e legazioni, Roma, Edizioni di Storia e Letteratura, p. 143176.

Gualdo, Riccardo (2017), L'italiano dei giornali, Roma, Carocci (I ed. 2007).

Koch, Peter e Wulf Oesttereicher (1997), «Zur Fundierung von Diskurstraditionen», in Barbara Frank, Thomas Haye e Doris Tophinke (ed.), Gattungen mittelalterlicher Schriftlichkeit, Tübingen, 1997, p. 19-41. 
Mari, Michele (1988), «Tiraboschi e Bettinelli: un'amicizia erudita», Giornale Storico della Letteratura Italiana, vol. CLXV, n530, p. 228-279.

Mari, Michele (1990), «Le tre lliadi di Melchiorre Cesarotti», Giornale storico della letteratura italiana, vol. CLXVII, n539, p. 1-75.

Mari, Michele (2013), La critica letteraria nel Settecento, Milano, Ledizioni.

Masini, Andrea (1977), La lingua di alcuni giornali milanesi dal 1859 al 1865, Firenze, La Nuova Italia.

Matarrese, Tina (1993), /l Settecento, Bologna, il Mulino.

Merida, Raphael (2017), «Questioni intorno alla lingua nelle riviste letterarie del primo Settecento», Circula, n 5, p. 7-24.

Roggia, Carlo Enrico (2013), La lingua della poesia nell'età dell'illuminismo, Roma, Carocci.

Scavuzzo, Carmelo (1988), Studi sulla lingua dei quotidiani messinesi di fine Ottocento, Firenze, Olschki.

Schwarze, Sabine (2020), «“Il traduttore a chi legge”. La fenomenologia della prefazione alle traduzioni italiane del Settecento», inTRAlinea, numero speciale La traduzione e i suoi paratesti, disponibile su http://www.intralinea.org/specials/article/il_traduttore_a_chi_legge. [Sito consultato il 15 giugno 2020].

Serianni, Luca (1989), Storia della lingua italiana. Il primo Ottocento: dall'età giacobina all'Unità, Bologna, il Mulino.

Serianni, Luca (1998), «La lingua italiana dal cosmopolitismo alla coscienza nazionale», in Enrico Malato (ed.), Storia della letteratura italiana, vol. VI, Roma, Salerno, p. 187-237.

Serianni, Luca (2012), Italiano in prosa, Roma, Carocci.

Sironi, Francesco (2018), «La 'Batracomiomachia' di Alessandro Garioni tra greco, italiano e milanese», Italiano LinguaDue, vol. 10, n² 2, p. 310-318.

Wilhelm, Raymund (2005), «Diskurstraditionen», La lingua italiana, vol. I, p. 157-161. 